\title{
Towards Gadget-Free Internet Services: A Roadmap of the Naked World
}

\author{
Ijaz Ahmad ${ }^{a}$, Tanesh Kumar ${ }^{a}$, Madhusanka Liyanage ${ }^{a}$, Mika Ylianttila ${ }^{\mathrm{a}}$, Timo Koskela ${ }^{\mathrm{a}}$, Timo \\ Braysy $^{\mathrm{a}}$, Anttonen Antti ${ }^{\mathrm{b}}$, Pentikäinen Vesa ${ }^{\mathrm{b}}$, Soininen Juha-Pekka ${ }^{\mathrm{b}}$, Jyrki Huusko ${ }^{\mathrm{b}}$ \\ ${ }^{a}$ University of Oulu, Finland \\ ${ }^{b}$ VTT Technical Research Centre of Finland, Oulu, Finland
}

\begin{abstract}
This paper presents a roadmap for the transition from current gadget-centric digital services towards a gadget-free services environment called the Naked world. The main idea of the Naked world is that all the services which are currently provided by gadgets will be provided by the infrastructure, thus no gadgets will be needed to use any kind of digital services. When a user in the Naked world intends to use a service, the infrastructure senses the user, the nearby intelligent surrounding launches an interactive user interface, performs identification through biometric identities, provides the service, and then closes the session when the user finishes the job. Therefore, the Naked world comprises highly intelligent and context-aware interactive environments. The vision of the Naked world is an evolution towards a user-friendly and ubiquitously available digital services, which is naturally bounded by the technological advancement. Henceforth, this paper presents the essential technologies and functional requirements along with the current and forthcoming novel technological concepts and challenges for the realization of the Naked world.
\end{abstract}

\section{Introduction}

Since the invention of the computer, one main trend for its use has been to provide digital services that automate routines, simplify tasks, or just entertain the user. The technical inventions have miniaturized computers and created communication networks that connect computers together. We have experienced the transition from mainframes to PCs, laptops, smart phones, embedded computers, wearables, and other gadgets towards ubiquitous computing in general. At the network side we have seen similar evolution. Internet is no longer a network of computers. Currently we talk about the Internet of Things (IoT) [1] and semantic information-centric networks [2]. In the area of digital services, the focus has moved from applications and systems to ambient intelligence and smart environments that are built in mash-up principles, exploit massive amounts of collected information, and utilize shared and distributed resources.

This paper evaluates the vision of the Naked world that is under investigation in the Naked Approach Project [3]. The Naked world vision pushes the ubiquitous computing and ambient intelligence concepts technologically into extreme and extends those concepts with the vision of ubiquitous interaction into a

Email addresses: iahmad@ee.oulu.fi (Ijaz Ahmad), tanesh.kumar@ee.oulu.fi (Tanesh Kumar), lliyanag@ee.oulu.fi (Madhusanka Liyanage), over@ee.oulu.fi (Mika Ylianttila), timo.koskela@ee.oulu.fi (Timo Koskela), braysy@ee.oulu.fi (Timo Braysy), Antti.Anttonen@vtt.fi (Anttonen Antti), Vesa.Pentikainen@vtt.fi (Pentikäinen Vesa), Juha-Pekka.Soininen@vtt.fi (Soininen Juha-Pekka) 
gadget-free way of life, called the Naked world. By gadget, we mean any electronic tool or machine that has the capability to offer any digital service e.g. smart phones, laptops, tablet computers and smart wearable devices. The main idea of the Naked world concept is that any user can live "naked" which means that users can access digital services without having to carry or wear any personal gadgets. In the Naked world, services and User Interfaces (UIs) appear and become active from surrounding environment when needed, and disappear and become inactive when not needed, as shown in Fig. 1.

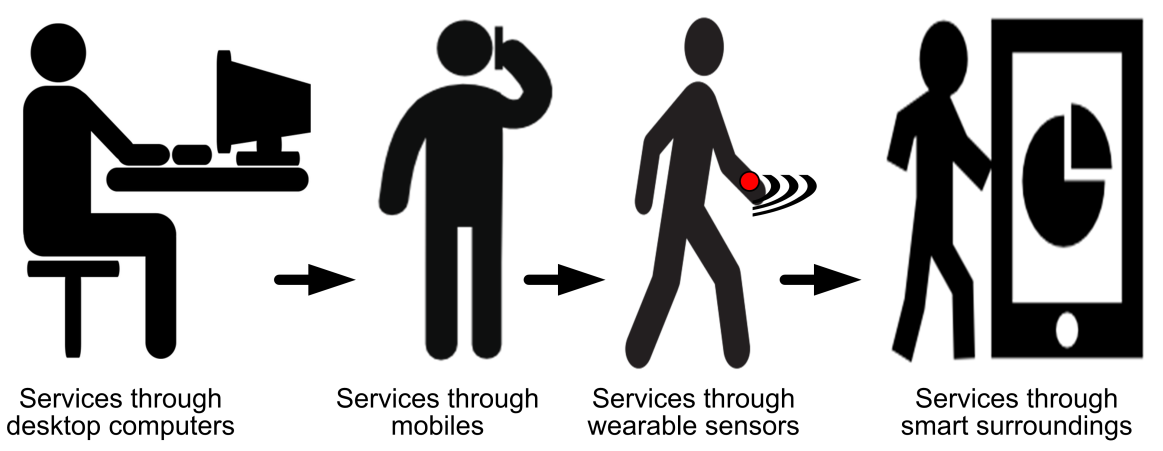

Figure 1: The transformation of digital services and modes of access towards the Naked world.

The Naked world vision is technically and socially a huge challenge. For example, it requires totally embedded user interaction infrastructure solutions available everywhere. Similarly, the Naked world requires new ways of offering services based on novel business models and collaboration among all the stakeholders including vendors, infrastructure owners, operators and users. Therefore, the Naked world vision may not be practical or realistic to our comprehension at this moment. However, in more limited spaces the benefits and value of some of its ideas and solutions have been demonstrated already by ambient intelligence and smart environments research [4]. Thus, the vision of the Naked world requires investigation of technologies and solutions needed for the transformation towards such an intelligent and context-aware environment. Before evaluating the potential architectural choices, we present a holistic view of different requirements supporting the vision of the Naked world. This leads us to a conceptual roadmap presenting how services will be moved to the infrastructure.

This paper presents some promising technological concepts and challenges in the scope of the Naked world. These technologies in principle support appearing/disappearing UIs, versatile services, storing and sharing of data and services, identification and authentication processes, and enabling connectivity between the entities and services. This paper is organized as follows. Section 2 describes the background of the evolution process towards the Naked world. In Section 3, the roadmap towards nakedness is introduced with a focus on how the services will be transferred to the infrastructure. Key requirements and generic architecture concepts of the Naked world are presented in Section 4 followed by discussion in Section 5. Section 6 concludes the paper. 


\section{Background}

The Internet has evolved from connecting only computers towards pervasive Internet of people and things [5], enabled by the underlying communication systems that evolved from a network-centric design paradigm towards a user-centric paradigm [6], [7]. Similarly, simple computing systems have evolved towards high-capacity and intelligent context-aware systems that understand the user behavior and environment, and offer diverse sets of services accordingly [5]. Consequently, the service providers using these technologies adopted new modes of service offerings not only to maximize their own benefits but to meet the user requirements of ease of use and costs. For instance, service providers offer higher storage and computing resources to their customers, thus relinquishing the need of user owned high capacity storage and computing resources.

The Naked world envisions even further technological development to enable an environment where users will be able to access and use all types of digital services without owning or carrying any type of computing or storage devices. This will also require new modes of service offering. For example, service providers will need to profile users more accurately not only by the physical demand attributes (e.g., speed, spatial distribution, device capabilities), but also by the physiological attributes (e.g., sensory response time, language difference) and personal attributes (e.g. content preference, service consumption habits, quality tolerance). However, such profiling will open the challenges of user privacy. Therefore, the Naked world requires dramatic development not only in the technology side, but also in terms of legislation and regulations to ensure user privacy.

To make the Naked world concept more comprehensible, consider a virtual environment in which users wear gadgets to sense or simulate their presence and interact with the environment. In Immersive Virtual Reality (IVR) [8] an environment is replicated and the users' physical presence interacting with the environment is simulated. Generation of perceptual illusions of ownership in IVR through a virtual body acting as the real body has already been proven [8]. The Naked world can be considered as a practical extension of IVR from virtual to a real environment through the technological development discussed in the following sections. However, unlike virtual environments, the Naked world will comprise highly context aware environments to offer services based on user needs and requirements.

Context is generally defined as any information that can be used to characterize the situation of a person, place or an object [9]. Context-awareness is described as the key requirement of pervasive computing systems to enable computing systems to respond and adjust itself to user needs [10]. The Naked world will require the computing systems embedded in the surrounding environment to be highly context aware to provide all the intended services ubiquitously. A generic context awareness roadmap for the Naked world is presented in Fig. 2. However, many challenges for enabling the context-aware environment need to be addressed, such as context information storage, sharing mechanisms, and privacy implications. Similarly, automation of user location tracking, identification, and service provision are required to enable the services to appear and disappear in different proximities and according to the user needs. In the following section, we describe the roadmap for the Naked world. 


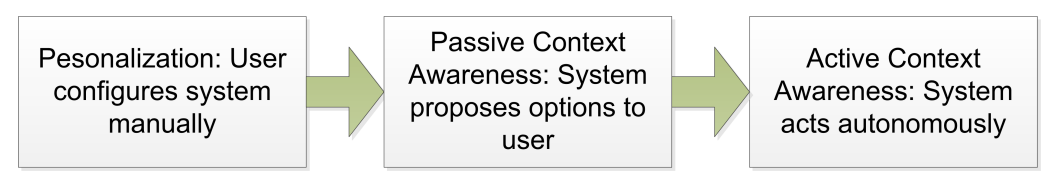

Figure 2: Evolution of context awareness.

\section{Roadmap Towards The Naked World}

The development towards the Naked world is not a simple or straightforward but a complex transition where development will occur in various technological dimensions such as computing, user-interaction, communication networks, data storage and sharing mechanisms, and security and privacy etc. Practically, the vision will be first experienced in limited smart spaces and later on such smart spaces will be connected to form a unified Naked world. In Table 1, we have listed few of the technologies to explain the transition process from the current gadgets-based services towards the Naked world in three phases. The phases of the transition are described below.

\subsection{The transition phases}

The transition towards the Naked world is mainly a transition of digital services from User Equipment (UE) to an ambient and intelligent environment that is naturally bounded by the evolution of the necessary technologies. Hence, the roadmap inherently includes the aspects of existing evolutionary steps, including mechanisms for data and applications transfer from the gadgets into the infrastructure that enable ubiquitous service availability; novel interaction mechanisms enabling user-infrastructure interaction; user identification so that the user is provided with personalized services by the infrastructure; and communication techniques or modalities that enable interaction between infrastructural elements e.g. for content sharing and traffic redirection with user mobility. The transition is pictorially presented in Fig. 3. To make it comprehensible, the transition is divided into three phases that starts from bearables, moves towards wearables, and then finally progress towards the last phase, i.e. nearables. In reality, the phases are overlapping but the development is not in the first phase in all the aspects as described below.

Services through bearables: This phase represents the current mainstream approach to obtain digital services through personal interaction devices. As in the current case the service subscriber carries mobile and handheld devices that include user identification and interface. The term bearables come from the Naked world vision that the users unwillingly use (bear) laptops, mobiles, tablets or other hand-held devices to access services. The Naked world assumes that users are not very comfortable to carry gadgets every time and everywhere to access diverse sets of services.

Services through wearables: This phase extends the use of wearable devices, termed wearables in this paper. Wearables are slowly and gradually replacing gadgets but the development of wearables is still in the early stages [11]. From the last decade wearable technology is improving and playing a vital role in healthcare, military and personal assistance [12], [13]. These devices are already available in different forms for example smart wristwatches, smart jackets, smart glasses, smart rings, etc. Improvements in miniaturization and better battery technologies made it possible to extend the wearable technology in all aspects of daily life [14]. IoT [15] and Wireless Sensor Networks (WSNs) [16] technologies are 


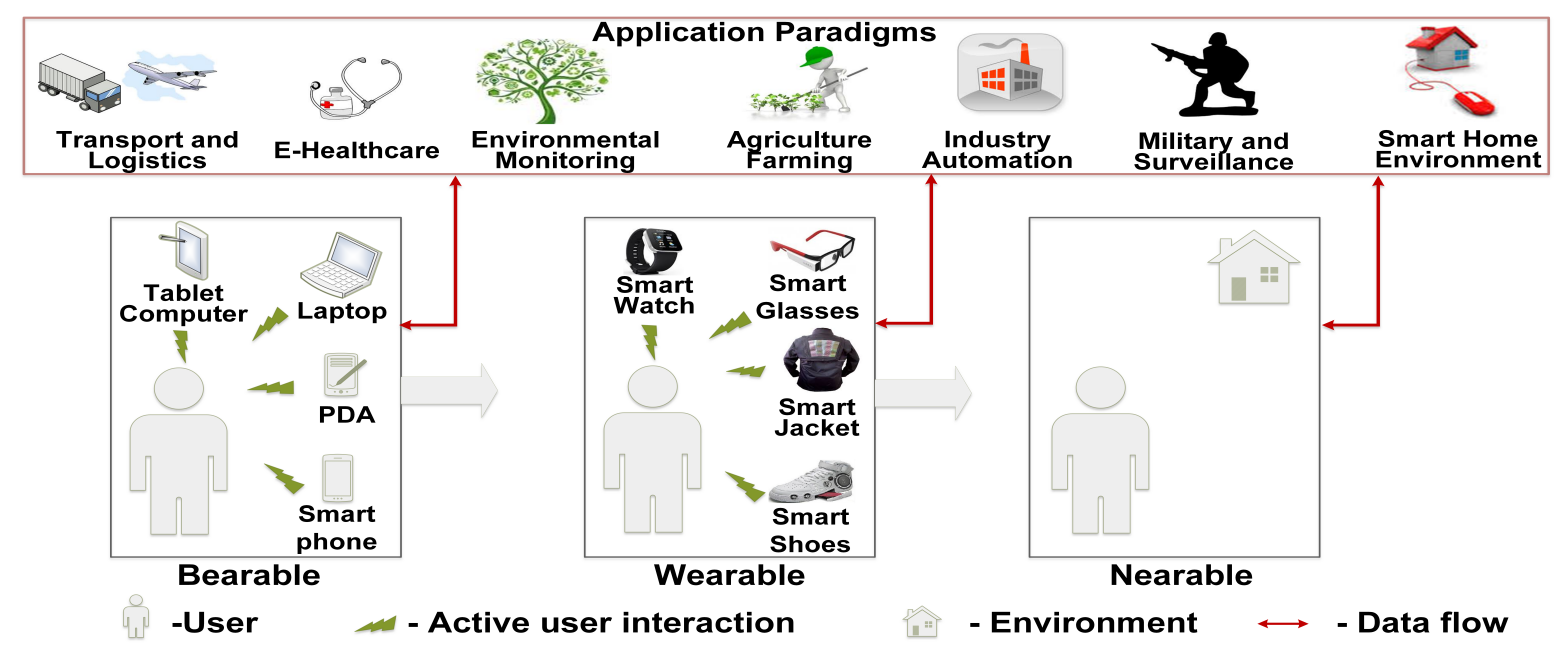

Figure 3: Phases of the transition process.

providing versatile options to enlarge the scope of wearable devices [17]. The integration of IoT into the mainstream communication technologies such as the 5th Generation (5G) wireless networks will enable novel services for wearables by providing connectivity to cloud-based services [18].

Services through nearables: This phase assumes that all the UIs, computing technologies and service provisioning mechanisms are embedded in the nearby surroundings, and thus such surroundings or environments are termed as nearables. Nearables have capabilities to detect a user, project UIs in front of the user, perform identification and then provide the required services according to the user profile. Furthermore, nearables are intelligent enough to react according to the user context and history to provide the intended services accordingly. Once the user finishes using services, the sessions will close and the UIs will disappear. Such nearables when connected together to provide services everywhere will fulfill the vision of the Naked world.

Below we describe the supporting technological concepts that are required for realizing the vision of the Naked world. These selected technologies are listed in Table 1.

Table 1: Example of transition of technologies towards the Naked world

\begin{tabular}{|l|c|c|c|}
\hline \multirow{2}{*}{$\begin{array}{l}\text { Selected } \\
\text { Technologies }\end{array}$} & \multicolumn{3}{|c|}{ Phases towards Nakedness } \\
\cline { 2 - 4 } Interaction & UE Interface & Wearable devices & Interactive UIs \\
\hline Service Logic & UE & Clouds & In surroundings \\
\hline Storage & Personal or Local & Shared & All stored in infra. \\
\hline Identification & UE Interface & Wearable & Via surroundings \\
\hline Communication & Fixed/Wireless & Dense/HetNets & Embedded in Infra. \\
\hline
\end{tabular}




\subsection{Gadget-free interaction}

The interaction in the Naked world happens without personal devices and directly with the environment. This transition will very likely happen stepwise from interaction through bearables to wearables and then nearables, which may overlap in time. The end goal is that interactive UIs appear in front of the user when he intends to use a service and fades away or go to background when the job is finished. The development in this direction is already happening. Interactive UIs in the form of smart tvs [19] is one such example. User authentication is carried out through face recognition and the tv programms can be controlled through hand gestures as described in [19]. Another example is the smart curtain [20] that interacts with users through touching and moving.

To enable ubiquitous availability of services, new advanced communication technologies will be required not only to connect smart objects in surrounding but also enable user and session mobility. For example, converged communication technologies [21] are already in use to keep different user-initiated session alive with user mobility. Converged networks have the capability to provide unrestricted combination of information and services from various domains such as video and audio streaming, web, IP television, sensors, RFIDs and online games [22]. Using such communication technologies the services will be able to provide an observable pleasing effect to users via sophisticated UIs, invisible and robust infrastructures which live along the subscriber needs.

\subsection{Transferring service logic from gadgets to infrastructure}

The service logic, i.e. applications, is moved from devices to the infrastructure that has UIs and highend computing devices. The infrastructure is normally owned or controlled by the service providers who provide different services based on user consent and business terms and conditions. While providing user data to the infrastructure, the infrastructure owner or service provider also ensure the user privacy and security requirements. This transition is already happening in the form of Software-as-a-Service (SaaS), Platform-as-a-Service (PaaS) [23], Infrastructure-as-a-Service (IaaS) [23]. A prominent example is the cloud based services [24] that host web-based applications such as Office 365, Google Docs, and online shopping websites paving the way towards the Naked world vision of having the service logic outside the gadgets. In such shared environments, security of user data will be a major concern. For example, multitenancy in shared environments bring about the challenges of eavesdropping, resource hijacking and stealing etc. Therefore, security and privacy must be ensured at all levels such as application level, operating system level and even infrastructure level [25].

Furthermore, context awareness will play a major role in timely service provisioning. The access to services must be provided for a specific context having the interactive environment being capable to understand the context in which the user wants to interact. Therefore, a paradigm shift is required from subject-centric access control to a context-centric one to enable ubiquitous service access [26].

\subsection{Storing and sharing data}

The data is moved from local storage or personal devices to the interactive infrastructure that will provide services. The infrastructure will use high-end data storage servers embedded into it or will be 
connected to cloud storage systems or data centers through ultra-high speed links. To enable ubiquitous access to services, the infrastructure contains UIs on one side and high-speed links to data storage systems on the other. Therefore, the interactive infrastructure has capability to share user information with other interfaces and infrastructure components with no observable delays. The main aim of the Naked world is to ensure availability of data and information to all the applications and services that the user can access anytime from anywhere. Thus, storage capacity building in or near the interactive infrastructure, and ultra-fast communication links between interfaces and storage systems are the main requirement of the Naked world.

\subsection{Identification}

Another functionality that will be moved from gadgets will be the identification process of the user. Moving from the current gadget-based username/password identification, wearables such as microchips implanted in the body and RFID tags [27] have gained attention for its use for identification purposes [17]. Biometric identities will be used for identification in the Naked world that will require physiological and behavioral characteristics including fingerprints, face recognition, hand geometry, voice, and retina and iris patterns [28]. Hence, the main challenge will be the detection of users' characteristics and their bio-signatures. Furthermore, the identification with biometric identities will be carried out by the infrastructure alone without active identity proofing by the user. One step already in this direction is identity proofing from facial recognition without active user involvement, for example monitoring of users in unconstrained environments [29], autonomous recognition from face in surveillance systems [30], and other approaches of passive facial recognition as described in [31].

\subsection{End-to-end communication}

The provisioning of services to users in any geographic location without gadgets requires highly robust, intelligent and adaptable communication infrastructure and architectures. Communication architectures in the Naked world seems to be highly complex due to large number of infrastructural elements needed to provide connectivity with high data-rates, high Quality of Service (QoS) and Quality of Experience (QoE), strict latency constraints and the huge number of IoT devices. Seemingly there will be service disruptions with user movement due to lack of always connected gadgets, although context-centric networking will enable service session migration using, for example, converged networking. Moreover, resource binding will be based on biometric identities rather than the IP or MAC address of a gadget.

\section{Architectural Requirements}

In this section, we describe the main technological or architectural disruption points and their impact on the infrastructure and system development in the path towards the Naked world. In the smart phone era where most of our daily routine functions are embedded in the gadget, moving towards a life without gadgets will not only be technologically demanding but socially challenging. Hence, the transition process will be a complex and multidimensional one making it difficult to model and present in few steps, also 


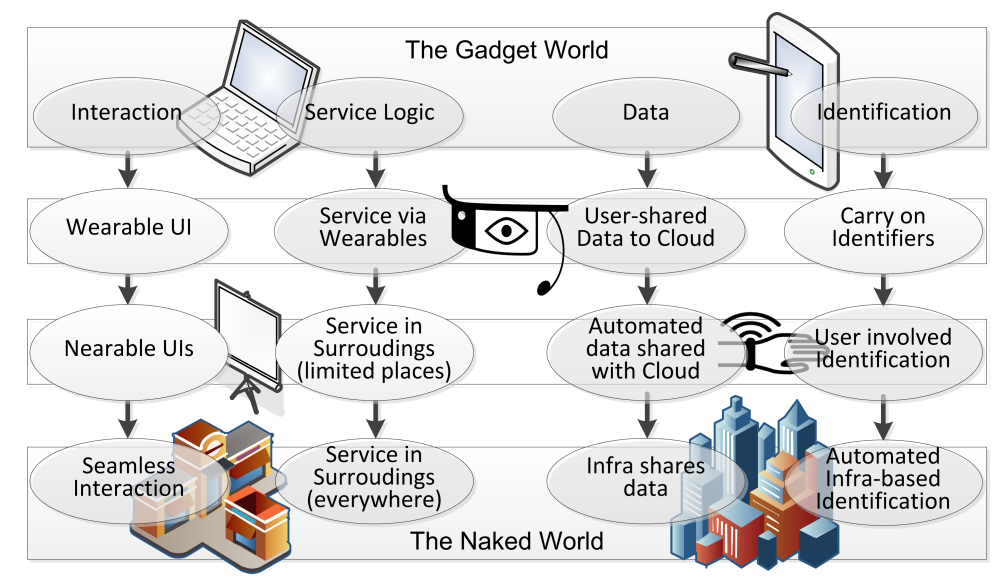

Figure 4: A view of multiple services in transformation from the gadget world to the Naked world.

depicted in Fig. 4. Hereby, we motivate future research work to conceptualize the vision and thus, try to outline the possible direction towards the realization of the Naked world.

A view of the technological dimensions given in Fig. 4 shows the complexity of technological convergence of gadget-based services to the Naked world. Table 2 presents the selected technologies with different enabling mechanisms required in each technology, and the corresponding challenges associated with each of them. The challenges presented against each mechanism are potential obstacles that must be resolved before their use in the Naked world. For example, in interaction if the challenge of latency in user detection is addressed, the process of serving a user will begin. However, until the user is detected no UI will appear to serve the user. Below we discuss these technological concepts and the challenges associated with them.

\subsection{Infrastructure for gadget-free interaction}

In the Naked world there will be a user-friendly natural interaction directly with the environment in which all the services will be embedded. Hence, the Naked world need to be highly context aware, adaptive and capable to observe user's activity to learn users' incentives, trigger service provisioning e.g. through interactive displays, and provide the required services and respective content. A feedback cycle must be initiated upon the user presence to adopt the interface and services accordingly. User interaction modalities have significantly changed from desktop interfaces towards user-friendly hand held and wearable devices.

The gadget-free interaction combines various technologies such as user detection or sensing technologies, context awareness to know the intended services that will be used, offering a user-friendly authentication mechanism e.g. recognition from face, voice or walking style, and providing the service through an interactive display. Human Computer Interaction (HCI) modalities have already progressed to enable interaction through physiological or biosignals, silent speech, air-writing, and eye gaze, etc. [32]. Conversational interfaces (talking bots) [33] and gesture-based interactions [34] have enabled users to directly talk to a system, and are capable to gather information or provide a multitude of services. Hence, from the interaction perspective we are already near the Naked world, though, in limited spaces. Hence, 
Table 2: Technological requirements for the selected enabling technologies

\begin{tabular}{|c|c|c|c|}
\hline Technology & Mechanisms & Required for & Challenges \\
\hline \multirow[t]{6}{*}{ Interaction } & \multicolumn{3}{|c|}{ Explanation: User will interact with appearing/disappearing UIs } \\
\hline & User detection & To offer services & Latency in user detection \\
\hline & UI setup & Window to services & Diverse ways to interact \\
\hline & UI flexibility & Need-based appearance & Omni-presence required \\
\hline & Identification & To authenticate & Identity matching dealy \\
\hline & Context awareness & Proactive service availability & Context differentiation \\
\hline \multirow[t]{4}{*}{ Service Logic } & \multicolumn{3}{|c|}{ Explanation: All the services processed in the nearby environment } \\
\hline & Edge or fog computing & Computing near user & Resource binding \\
\hline & Service migration & Services follow users & Latency \& context \\
\hline & Tracking Technology & User positioning & Track user without device \\
\hline \multirow[t]{4}{*}{ Storage } & \multicolumn{3}{|c|}{ Explanation: Data is stored and shared by the environment } \\
\hline & Storage in Clouds & Huge data volume & Privacy \\
\hline & Data transport & Saving \& sharing data & Latency \& high speed \\
\hline & Scalability & High availability & Frequent sharing \\
\hline \multirow[t]{5}{*}{ Identification } & \multicolumn{3}{|c|}{ Explanation: Users identified and authenticated without user-owned devices } \\
\hline & Biometric identification & Authentication \& access & Matching delays \\
\hline & Access control & Legitimate use & Accuracy, latency \\
\hline & Bio Scanning & Identity proofing & Identify user without device \\
\hline & Service anchoring & Biometric id based & Session migration \\
\hline \multirow[t]{5}{*}{ Communication } & \multicolumn{3}{|c|}{ Explanation: Flexible, adaptable and agile, and secure communication architecture } \\
\hline & Software-based & Higher flexibility & Malware attacks \\
\hline & Virtualized & Resource sharing & Service binding \\
\hline & End-to-end security & User/infrastructure security & Mult-faceted vulnerabilities \\
\hline & Privacy & Ensure user privacy & Identity \& location privacy \\
\hline
\end{tabular}

the main challenge is to extend the availability of such interaction mechanisms from limited spaces to the omni-present one as envisioned by the Naked world.

\subsection{Infrastructure for service logic provisioning}

The Naked world will enable the phenomenon of Surrounding as a Service in which the surrounding environment will tune itself for providing user-intended services. Since all the services that are performed by the currently used gadgets e.g. smart phones, need to be converged into an interactive environment, the computing burden will shift to the devices embedded in the infrastructure. Cloud, edge and fog computing have already been used to perform computing in the infrastructure [35]. Hence, computing through a shared pool of centralized and decentralized resources i.e. mix of IoT, edge devices, and centralized clouds will be the key requirement of the Naked world. In practice, the Naked world will 


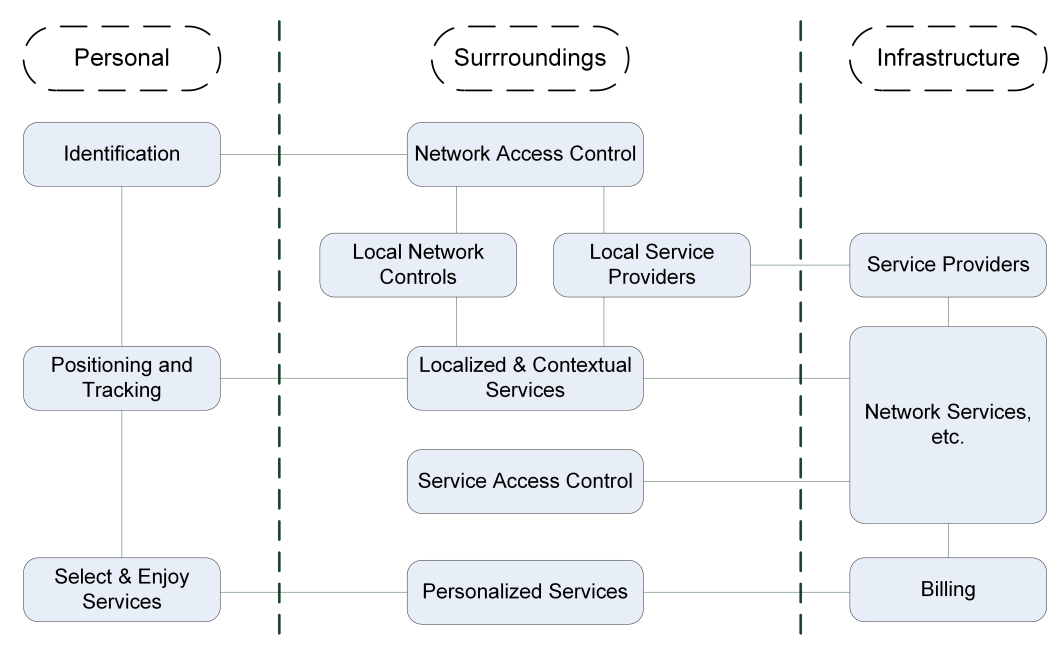

Figure 5: Elements for service provisioning.

be the realization of the famous John Gage's phrase, "the network is the computer" [36], since all the processing will be carried within the network of connected processing devices.

The main technological challenges will be related to user positioning and context awareness. For example, it will be difficult to position a user having no connected devices or digital implants. Similarly, the context need to include current and possible future location to enable the intended services at various visiting locations. However, advanced intelligent services and systems, e.g. cognitive systems [37], will be able to learn individual user practices and needs, and thus will be able to offer services according to the context. Fig. 5 presents the most relevant technologies that are required for the architecture from the perspectives of services in personal spaces, surroundings and the infrastructure which control the service provisioning. The possible logical connections among the service logic provisioning elements are indicated by the connecting lines in Fig. 5. The following service provisiong elements are necessary to satisfy user service requirements:

- User identification: To enable service access while satisfying the security and privacy requirements.

- Positioning: To provide location based user intended services and enable service migration with user mobility.

- Context Awareness: To sense the need and nature of the intended services by the user and adapt the environment accordingly.

- Personalized services: To enable service movement with the user according to his needs and QoS and QoE profile.

- Interactive Interfaces: To provide the multi-model interactivity to the user. The interfaces appear and disappear according to the need.

\subsection{Infrastructure for storing and sharing data}

In the Naked world, the data is stored in the infrastructure and shared among infrastructural elements for higher availability, quick service access, and context awareness. The process of storing and sharing 
data in the infrastructure such as clouds has already started in various forms of online services in which users either deliberately store and share data e.g. social media, or, are required to provide their data e.g. in modern consumer services. In the Naked world, automated devices and machines will start to create digital representation of everything in real time which will mean that everything that exist in realworld can be analyzed and used globally by digitally controlled systems. There are already examples of such digital representation in the form of video surveillance and security solutions that try to recognize people from faces, identify possible threats, and share the information with the help of low powered IoT devices [38].

The cloud is understood as a monolithic abstraction, but the amount of data and the performance requirements of applications will force us to take into account other realities such as distribution of data. The current trend of moving computation closer to network edges will become more important, because real-time responses are otherwise impossible to achieve due to fundamental limits of communication speed [39]. Therefore, the concepts such as fog [40] [41] and mobile edge computing [42] [39] will play a major role in the Naked world. Similarly, concepts such as artificial intelligence and big data analytics [43] will provide more powerful tools for understanding and profiling of users' needs and behaviors, and gather and share data proactively whenever needed. However, security, privacy, and access control of data will be major challenges in the Naked world. These issues will require strict regulations and legislation besides technological developments.

\subsection{Infrastructure for identification}

The identification technologies are already evolving from usernames and passwords to ID tags to bio-signatures such as fingerprints, face and retina scanning [44] [45]. Therefore, biometric is the natural and potential candidate for the user's identification in the Naked world [46]. Table 3 outlines various performance metrics for biometric identification systems along with their acceptability indicators and potential challenges for their use in the Naked world. The challenge is that we have to reduce the False Acceptance Rate (FAR), False Rejection Rate (FRR) and increase the accuracy for the Naked world. A combination of biometric modalities such as face and voice or face and fingerprint etc. can also be used depending on the situation and user willingness. Initially, users will actively participate in the identification process, e.g. to provide their fingerprints, voice or retina scanning for using services. Later on, we have to omit the user involvement in identification for accessing services. The ways in which someone may be identified fall into three categories, i.e. the knowledge factors (something the user knows), the ownership factors (something the user has) and the inherence factors (something the user is or does). In the Naked world, inherence factors will be mainly used as the identifiers of users.

However, biometric identities are vulnerable to theft [47]. For example, face and voice biometrics can be easily captured without the user consent. Therefore, new mechanisms that bind users in real-time with their identities will be required. Furthermore, IP addresses are used to identify gadgets over the network, however, users in the Naked world will be recognized through biological information that the users have. Hence, a major challenge will be to provide services to users through biological identification mechanisms since human beings may not hold IPs. The identification system and mechanisms must also 
be fast enough to avoid the chances of session break ups due to delay in the process.

Table 3: Metrics for biometric identification mechanisms [48], [49], [28]

\begin{tabular}{|l|c|c|c|c|l|}
\hline \hline Biometric Traits & FAR & FRR & Accuracy & Acceptability & Challenges \\
\hline Face & $1 \%$ & $10 \%$ & $94.10 \%$ & High & Varied light \\
\hline Fingerprint & $2 \%$ & $2 \%$ & $94.70 \%$ & Medium & Distortion \\
\hline Iris & $0.94 \%$ & $0.99 \%$ & 94.40 & Low & Low light \\
\hline Voice & $2 \%$ & $10 \%$ & $90 \%$ & High & Multilingual \\
\hline $\begin{array}{l}\text { Multi(Face,Iris } \\
\text { Fingerprint ) }\end{array}$ & $0.11 \%$ & $0.08 \%$ & $96.5 \%$ & NA & $\begin{array}{l}\text { Latency, high } \\
\text { complexity }\end{array}$ \\
\hline
\end{tabular}

\subsection{Infrastructure for communication}

In the Naked world, the communication infrastructure will be integrated or merged into the surrounding infrastructure, and thus will not be visible to user yet ensure ubiquitous availability and high performance with low costs and high energy efficiency. Hence, the network will require high level of automation, context awareness, flexibility and adaptability to automatically shrink or grow. The network must also adjust all the parameters, such as QoS and QoE, to respond to the user and services needs. Therefore, programmable networking technologies that enable run-time live service migration, network resource sharing and optimization with agility and adaptability will be needed. Furthermore, distributed computation technologies that perform fast computing and processing near the user or the network edge need to be coupled with the networking technologies.

Communication networks are already evolving and new technologies are incorporated as shown in Fig. 6. This trend of new technologies will most likely continue to meet the requirements of the Naked world. The main challenges will be related to flexible network operation to keep up with the extreme traffic demands and fulfill the performance, portability, elasticity, energy efficiency, and CapEx and OpEx requirements. Network function softwarization(e.g. Software Defined Networking (SDN) [50] [51]) and virtualization [52], mobile clouds [53], and mobile edge and fog computing [40] [54] will play a key role in flexible network operations. Similarly, context aware networking [55] combined with novel access and transport technologies [56] [57] using the concepts of converged networking [22] will meet the communication requirements of the Naked world.

\section{Discussion}

The Naked world vision will empower the users to get rid of carrying gadgets everywhere, yet use the same or even advanced services through highly intelligent and interactive context aware environment. Hence, the Naked world comprises complex infrastructures simply because the tasks that have been carried out with gadgets such as information collection, processing, storing, and interaction will be done by the systems integrated in the infrastructure. New types of services will come into play along with the deployment of such infrastructures that will open new business opportunities. However, the success 


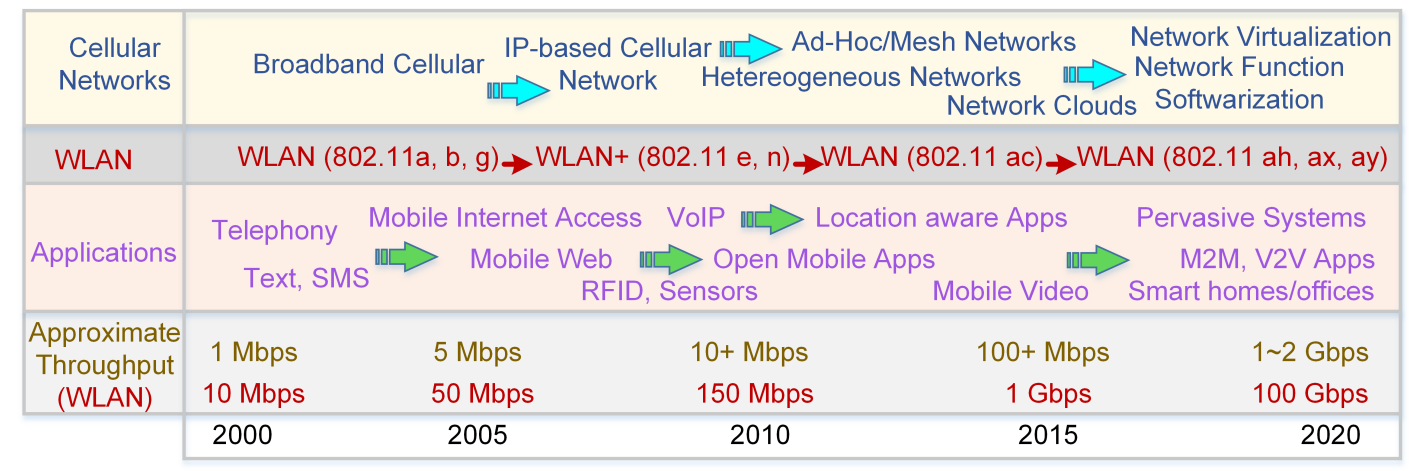

Figure 6: Evolution of communication networks.

of the Naked world will be highly dependent on user acceptability due to the required user trust and acceptability. Thus, the realization of the Naked world will be challenging not only from technological point of view but also from business perspectives and social mindset.

The technological development towards the Naked world is already happening in the selected enabling technologies in constraint environments such as interaction in augmented reality, shared service logic and storage in cloud environments, automatic identification from face, and context aware and converged networking as described throughout the paper. However, there are many obstacles to overcome for their actual use in the Naked world. For example, providing interactive UIs everywhere the user may intend to use a service; security and privacy challenges in shared processing and storage environments; automatic detection and identification of huge number of users without gadgets; and context-aware service mobility. Security will be a major challenge since the whole infrastructure from UIs to communication links will be shared among a huge number of involved stakeholders. However, security-by-design approach must be considered to avoid the possible migration of the current security threats into the Naked world. Furthermore, it is highly probable that many new challenges will emerge with the practical realization of the Naked world.

The Naked world will provide a different business eco-system as compared to the current one. Currently, it is possible that a single service provider owns or manages all the resources in the infrastructure for various services. The Naked world, on the other hand, will be a huge pool of shared resources in a connected infrastructure that will provide services anywhere and anytime. Therefore, the infrastructure will be managed and controlled by different stakeholders such as operators, services providers, vendors and customers or users. Such eco-systems will require strong regulatory bodies to control and protect the rights of all the stakeholders. Furthermore, this vision will generate new business opportunities for all the stakeholders due to massive computing and storage capabilities, and fast and reliable communication systems connecting all the resources into a unified infrastructure to increase their availability for services everywhere.

It will be highly challenging to provide full privacy and anonymity in the Naked world. For example, currently most of the user data is stored in gadgets that can be visible only to the respective user. Similarly, the services can be accessed through interfaces in personal gadgets. However, all the data in the Naked world will be stored in the infrastructure, and the services can only be accessed through UIs in 
the infrastructure that the users will not be able to confine to himself only. An interesting approach for personalizing the UIs will be that the UI projects only in user-specified or private spaces e.g. his hands. Moreover, strong privacy measures will increase social acceptance of the Naked world. The user also needs to be aware of the security and privacy implications when he provides his data or uses particular services. The legal and regulatory bodies must also play their due roles for ensuring that the user and service providers abide by the mutual agreements.

\section{Conclusion}

The Naked world vision is: using all kinds of digital services without carrying personal gadgets. The Naked world infrastructure provides interactive interfaces that pop-ups when the user comes into its vicinity, provide access to all the intended services while maintaining privacy, and immediately go to background or exit as the user stops using it. Since the idea of the Naked world is a far-sighted userfriendly and high-tech digital society, the transition is naturally bounded by evolution of technologies that are needed to implement it. Therefore, this paper highlights the roadmap based on the current and forthcoming technological concepts, their challenges, and a way-ahead towards the Naked world. The main technological challenges revolve around the areas of computing, interactive interfaces, storage and sharing of data, user identification and privacy, and communication networks. To enable the Naked world, we must seek new technological concepts and ideas to address these challenges. In the nutshell, the infrastructure must be agile and adaptable, computationally powerful, intelligent, highly secure and use novel storage and communication mechanisms to provide user services with no observable delays.

\section{Acknowledgment}

This work has been supported by TEKES under project The Naked Approach Nordic perspective to gadget-free hyper connected environments. The authors would like to acknowledge the contributions of their colleagues in the project.

\section{References}

[1] M. R. Palattella, M. Dohler, A. Grieco, G. Rizzo, J. Torsner, T. Engel, L. Ladid, Internet of Things in the 5G Era: Enablers, Architecture, and Business Models, IEEE Journal on Selected Areas in Communications 34 (3) (2016) 510-527. doi:10.1109/JSAC.2016.2525418.

[2] G. Xiao, J. Guo, Z. Gong, R. Li, Semantic input method of Chinese word senses for semantic document exchange in e-business, Journal of Industrial Information Integration 3 (2016) $31-36$. doi:http://dx.doi.org/10.1016/j.jii.2016.07.002.

[3] J. Aikio, V. Pentikäinen, J. Häikiö, J. Häkkilä, A. Colley, On the road to digital paradise: The Naked Approach (2016).

URL http://www.nakedapproach.fi

[4] P. Remagnino, G. L. Foresti, T. Ellis, Ambient intelligence: a novel paradigm, Springer, 2005. 
[5] C. Perera, A. Zaslavsky, P. Christen, D. Georgakopoulos, Context Aware Computing for The Internet of Things: A Survey, IEEE Communications Surveys Tutorials 16 (1) (2014) 414-454. doi:10.1109/SURV.2013.042313.00197.

[6] K. Crisler, M. Anneroth, A. Aftelak, P. Pulil, The human perspective of the wireless world, Computer Communications 26 (1) (2003) 11 - 18. doi:http://dx.doi.org/10.1016/S1403-3664(02)00114-7.

[7] G. Liu, 5G-user centric network, in: 2015 Asia-Pacific Microwave Conference (APMC), Vol. 1, 2015, pp. 1-1. doi:10.1109/APMC.2015.7411348.

[8] K. Kilteni, I. Bergstrom, M. Slater, Drumming in Immersive Virtual Reality: The Body Shapes the Way We Play, IEEE Transactions on Visualization and Computer Graphics 19 (4) (2013) 597-605. doi:10.1109/TVCG.2013.29.

[9] A. K. Dey, Understanding and using context, Personal Ubiquitous Comput. 5 (1) (2001) 4-7. doi:10.1007/s007790170019.

[10] M. Satyanarayanan, Pervasive computing: vision and challenges, IEEE Personal Communications 8 (4) (2001) 10-17. doi:10.1109/98.943998.

[11] N. D. Heintzman, A Digital Ecosystem of Diabetes Data and Technology: Services, Systems, and Tools Enabled by Wearables, Sensors, and Apps, Journal of Diabetes Science and Technology 10 (1) (2016) 35-41. arXiv:http://dst.sagepub.com/content/10/1/35.full.pdf+html, doi:10.1177/1932296815622453.

[12] J. W. Cheng, H. Mitomo, The underlying factors of the perceived usefulness of using smart wearable devices for disaster applications, Telematics and Informatics 34 (2) (2017) 528 - 539. doi:http://dx.doi.org/10.1016/j.tele.2016.09.010.

[13] J. Pascoe, Adding generic contextual capabilities to wearable computers, in: Wearable Computers, 1998. Digest of Papers. Second International Symposium on, 1998, pp. 92-99. doi:10.1109/ISWC.1998.729534.

[14] A. Lymberis, Smart wearables for remote health monitoring, from prevention to rehabilitation: current R D, future challenges, in: Information Technology Applications in Biomedicine, 2003. 4th International IEEE EMBS Special Topic Conference on, 2003, pp. 272-275. doi:10.1109/ITAB.2003.1222530.

[15] F. Liu, C.-W. Tan, E. T. Lim, B. Choi, Traversing knowledge networks: an algorithmic historiography of extant literature on the Internet of Things (IoT), Journal of Management Analytics 0 (0) (0) 1-32. doi:10.1080/23270012.2016.1214540.

[16] H. Yan, L. D. Xu, Z. Bi, Z. Pang, J. Zhang, Y. Chen, An emerging technology - wearable wireless sensor networks with applications in human health condition monitoring, Journal of Management Analytics 2 (2) (2015) 121-137. doi:10.1080/23270012.2015.1029550. 
[17] L. D. Xu, W. He, S. Li, Internet of Things in Industries: A Survey, IEEE Transactions on Industrial Informatics 10 (4) (2014) 2233-2243. doi:10.1109/TII.2014.2300753.

[18] L. Jiang, L. D. Xu, H. Cai, Z. Jiang, F. Bu, B. Xu, An IoT-Oriented Data Storage Framework in Cloud Computing Platform, IEEE Transactions on Industrial Informatics 10 (2) (2014) 1443-1451. doi:10.1109/TII.2014.2306384.

[19] S. H. Lee, M. K. Sohn, D. J. Kim, B. Kim, H. Kim, Smart tv interaction system using face and hand gesture recognition, in: 2013 IEEE International Conference on Consumer Electronics (ICCE), 2013, pp. 173-174. doi:10.1109/ICCE.2013.6486845.

[20] T. Takashina, K. Aoki, A. Maekawa, C. Tsukamoto, H. Kawai, Y. Yamariku, K. Tsuruta, M. Shimokawa, Y. Kokumai, H. Koike, Smart curtain as interactive display in living space, in: SIGGRAPH Asia 2015 Posters, SA '15, ACM, New York, NY, USA, 2015, pp. 32:1-32:1. doi:10.1145/2820926.2820971.

URL http://doi.acm.org/10.1145/2820926.2820971

[21] A. Saleh, J. Borger, N. Raman, Converged communication services and competition in consumer markets, in: Networks 2008 - The 13th International Telecommunications Network Strategy and Planning Symposium, Vol. Supplement, 2008, pp. 1-10. doi:10.1109/NETWKS.2008.6231317.

[22] B. Aïdan, R. B. Hull, Applications for the converged communications world: Challenges and emerging technologies, Bell Labs Technical Journal 13 (2) (2008) 1-4. doi:10.1002/bltj.20295.

[23] V. Gonçalves, P. Ballon, Adding value to the network: Mobile operators' experiments with softwareas-a-service and platform-as-a-service models, Telematics and Informatics 28 (1) (2011) 12 - 21, mobile Service Architecture and Middleware. doi:http://dx.doi.org/10.1016/j.tele.2010.05.005.

[24] M. Armbrust, A. Fox, R. Griffith, A. D. Joseph, R. Katz, A. Konwinski, G. Lee, D. Patterson, A. Rabkin, I. Stoica, M. Zaharia, A view of cloud computing, Commun. ACM 53 (4) (2010) 50-58. doi:10.1145/1721654.1721672.

[25] L. Rodero-Merino, L. M. Vaquero, E. Caron, A. Muresan, F. Desprez, Building safe paas clouds: A survey on security in multitenant software platforms, Computers Security 31 (1) (2012) 96 - 108. doi:http://dx.doi.org/10.1016/j.cose.2011.10.006.

URL http://www.sciencedirect.com/science/article/pii/S0167404811001313

[26] A. Corradi, R. Montanari, D. Tibaldi, Context-based access control for ubiquitous service provisioning, in: Computer Software and Applications Conference, 2004. COMPSAC 2004. Proceedings of the 28th Annual International, 2004, pp. 444-451 vol.1. doi:10.1109/CMPSAC.2004.1342877.

[27] A. Juels, RFID security and privacy: a research survey, IEEE Journal on Selected Areas in Communications 24 (2) (2006) 381-394. doi:10.1109/JSAC.2005.861395.

[28] A. K. Jain, A. Ross, S. Prabhakar, An introduction to biometric recognition, IEEE Transactions on Circuits and Systems for Video Technology 14 (1) (2004) 4-20. doi:10.1109/TCSVT.2003.818349. 
[29] J. N. Worsey, Face recognition in an unconstrained environment for monitoring student attendance.

[30] S. Mittal, J. K. Rai, Wadoro: An autonomous mobile robot for surveillance, in: 2016 IEEE 1st International Conference on Power Electronics, Intelligent Control and Energy Systems (ICPEICES), 2016, pp. 1-6. doi:10.1109/ICPEICES.2016.7853652.

[31] X. Zhang, Y. Gao, Face recognition across pose: A review, Pattern Recognition 42 (11) (2009) 2876 - 2896. doi:http://dx.doi.org/10.1016/j.patcog.2009.04.017.

[32] H. P. D. Silva, S. Fairclough, A. Holzinger, R. Jacob, D. Tan, Introduction to the special issue on physiological computing for human-computer interaction, ACM Trans. Comput.-Hum. Interact. 21 (6) (2015) 29:1-29:4. doi:10.1145/2688203.

[33] L. C. Klopfenstein, S. Delpriori, S. Malatini, A. Bogliolo, The rise of bots: A survey of conversational interfaces, patterns, and paradigms, in: Proceedings of the 2017 Conference on Designing Interactive Systems, DIS '17, ACM, New York, NY, USA, 2017, pp. 555-565. doi:10.1145/3064663.3064672.

[34] S. S. Rautaray, A. Agrawal, Vision based hand gesture recognition for human computer interaction: a survey, Artificial Intelligence Review 43 (1) (2015) 1-54. doi:10.1007/s10462-012-9356-9.

[35] P. Garcia Lopez, A. Montresor, D. Epema, A. Datta, T. Higashino, A. Iamnitchi, M. Barcellos, P. Felber, E. Riviere, Edge-centric computing: Vision and challenges, SIGCOMM Comput. Commun. Rev. 45 (5) (2015) 37-42. doi:10.1145/2831347.2831354.

URL http://doi.acm.org/10.1145/2831347.2831354

[36] G. Author, The Network is the Computer -still.

URL https://blogs .oracle.com/vreality/the-network-is-the-computer-still

[37] P. Langley, J. E. Laird, S. Rogers, Cognitive architectures: Research issues and challenges, Cognitive Systems Research 10 (2) (2009) 141 - 160. doi:http://dx.doi.org/10.1016/j.cogsys.2006.07.004.

URL http://www.sciencedirect.com/science/article/pii/S1389041708000557

[38] J. Mao, Q. Zhou, M. Sarmiento, J. Chen, P. Wang, F. Jonsson, L. Xu, L. Zheng, Z. Zou, A hybrid reader tranceiver design for industrial internet of things, Journal of Industrial Information Integration 2 (2016) 19 - 29. doi:http://dx.doi.org/10.1016/j.jii.2016.05.001.

[39] M. T. Beck, M. Werner, S. Feld, S. Schimper, Mobile edge computing: A taxonomy, in: Proc. of the Sixth International Conference on Advances in Future Internet, Citeseer, 2014.

[40] F. Bonomi, R. Milito, J. Zhu, S. Addepalli, Fog Computing and Its Role in the Internet of Things, in: Proceedings of the First Edition of the MCC Workshop on Mobile Cloud Computing, MCC '12, ACM, New York, NY, USA, 2012, pp. 13-16. doi:10.1145/2342509.2342513.

[41] S. Yi, C. Li, Q. Li, A Survey of Fog Computing: Concepts, Applications and Issues, in: Proceedings of the 2015 Workshop on Mobile Big Data, Mobidata '15, ACM, New York, NY, USA, 2015, pp. 37-42. doi:10.1145/2757384.2757397. 
[42] Y. C. Hu, M. Patel, D. Sabella, N. Sprecher, V. Young, Mobile Edge Computing-A Key Technology Towards 5G, ETSI White Paper 11.

[43] A. Gandomi, M. Haider, Beyond the hype: Big data concepts, methods, and analytics, International Journal of Information Management 35 (2) (2015) 137 - 144. doi:http://dx.doi.org/10.1016/j.ijinfomgt.2014.10.007.

[44] W. B. Committee, et al., Biometric recognition: challenges and opportunities, National Academies Press, 2010.

[45] K. W. Bowyer, K. Chang, P. Flynn, A survey of approaches and challenges in 3D and multi-modal $3 \mathrm{D}+2 \mathrm{D}$ face recognition, Computer Vision and Image Understanding 101 (1) (2006) 1 - 15. doi:http://dx.doi.org/10.1016/j.cviu.2005.05.005.

[46] T. Kumar, M. Liyanage, A. Braeken, M. Ylianttila, Identity privacy preserving biometric based authentication scheme for naked healthcare environment, ICC, 2017.

[47] A. Alterman, "A piece of yourself": Ethical issues in biometric identification, Ethics and Information Technology 5 (3) (2003) 139-150. doi:10.1023/B:ETIN.0000006918.22060.1f.

[48] D. Bhattacharyya, R. Ranjan, F. Alisherov, M. Choi, et al., Biometric authentication: A review, International Journal of u-and e-Service, Science and Technology 2 (3) (2009) 13-28.

[49] S. Sarhan, S. Alhassan, S. Elmougy, Multimodal biometric systems: A comparative study, Arabian Journal for Science and Engineering 42 (2) (2017) 443-457. doi:10.1007/s13369-016-2241-0.

URL http://dx.doi.org/10.1007/s13369-016-2241-0

[50] M. Liyanage, A. Gurtov, M. Ylianttila, Software Defined Mobile Networks (SDMN): Beyond LTE Network Architecture, John Wiley \& Sons, 2015.

[51] I. Ahmad, S. Namal, M. Ylianttila, A. Gurtov, Security in Software Defined Networks: A Survey, IEEE Communications Surveys Tutorials 17 (4) (2015) 2317-2346. doi:10.1109/COMST.2015.2474118.

[52] N. M. K. Chowdhury, R. Boutaba, A survey of network virtualization, Computer Networks 54 (5) (2010) 862 - 876. doi:http://dx.doi.org/10.1016/j.comnet.2009.10.017.

[53] N. Fernando, S. W. Loke, W. Rahayu, Mobile cloud computing: A survey, Future Generation Computer Systems 29 (1) (2013) 84 - 106, including Special section: AIRCC-NetCoM 2009 and Special section: Clouds and Service-Oriented Architectures. doi:http://dx.doi.org/10.1016/j.future.2012.05.023.

[54] T. H. Luan, L. Gao, Z. Li, Y. Xiang, L. Sun, Fog computing: Focusing on mobile users at the edge, CoRR abs/1502.01815. 
[55] P. Mendes, Combining data naming and context awareness for pervasive networks, Journal of Network and Computer Applications $50 \quad(2015) \quad 114 \quad-\quad 125$. doi:http://dx.doi.org/10.1016/j.jnca.2014.09.015.

[56] P. Rost, C. J. Bernardos, A. D. Domenico, M. D. Girolamo, M. Lalam, A. Maeder, D. Sabella, D. Wübben, Cloud technologies for flexible $5 \mathrm{G}$ radio access networks, IEEE Communications Magazine 52 (5) (2014) 68-76. doi:10.1109/MCOM.2014.6898939.

[57] C.-L. I, C. Rowell, S. Han, Z. Xu, G. Li, Z. Pan, Toward green and soft: a 5G perspective, Communications Magazine, IEEE 52 (2) (2014) 66-73. doi:10.1109/MCOM.2014.6736745. 